\title{
Redefining Smoldering Myeloma
}

\author{
Dr. Amro El-Ghammaz \\ Lecturer of Internal Medicine, Hematology and Bone Marrow \\ Transplantation \\ Hematology and Bone Marrow transplantation unit, \\ Internal Medicine department, Faculty of Medicine, \\ Ain Shams University
}

Introduction:

Approximately $8 \%$ to $20 \%$ of patients with MM are recognized by chance without significant symptoms. Smoldering multiple myeloma (SMM) was initially recognized in the 1980s [1]. It bridged the gap between monoclonal gammopathy of undetermined significance (MGUS; a mostly premalignant disorder) and active multiple myeloma (MM). Until the International Myeloma Working Group (IMWG) classification system was developed, definitions had varied [2]. The common theme across studies was the universal recognition that there were asymptomatic patients who exceeded the limits of the definition of MGUS, who could remain without end-organ damage for years, and who outsurvived MM patients.

\section{Definitions of SMM:}

Kyle and Greipp first used the term "smoldering myeloma" in 1980 [1]. This expression referred to those patients who satisfied the following criteria: (1) M protein $>30 \mathrm{~g} / \mathrm{L}$, (2) bone marrow plasmacytosis $>10 \%$, (3) no end-organ damage, and (4) no progression of disease at 5 years. At the same time, Alexanian et al explored the use of the term "indolent" myeloma (IMM), an entity that allowed for up to 3 lytic bone lesions, a minimum bone marrow plasmacytosis of $15 \%$, and distinct minimum and maximum thresholds for immunoglobulin $\mathrm{G}(\lg G)$ and $\lg A$ [3]. Over time, Alexanian et al also separated the SMM (or asymptomatic MM) from the IMM, assigning a maximum value of M-spike of $45 \mathrm{~g} / \mathrm{L}$ for the former category [4]. Subsequent to the SMM and the IMM classifications came that of evolving MM, which was defined as an $M$ protein that abruptly increases when symptomatic MM develops [5]. It was not until 2003, when there was international consensus about the definitions of plasma cell disorders, that the criteria for SMM were established [2]. The international myeloma working group (IMWG) defined SMM as presence of bone marrow plasmacytosis $\geq 10 \mathrm{gm} / \mathrm{dL}$ (Figure 1) or $\geq 30 \mathrm{~g} / \mathrm{L} \mathrm{M}$-protein (Figure 2) and absence of: high calcium, hemoglobin $2 \mathrm{~g} / \mathrm{dL}$ below normal or $<10 \mathrm{~g} / \mathrm{dL}$, 
lytic bone lesions or osteoporosis with compression fracture, symptomatic hyperviscosity, amyloidosis, or $>2$ bacterial infections/12 months. Most subsequent publications have used this more uniform definition [6].

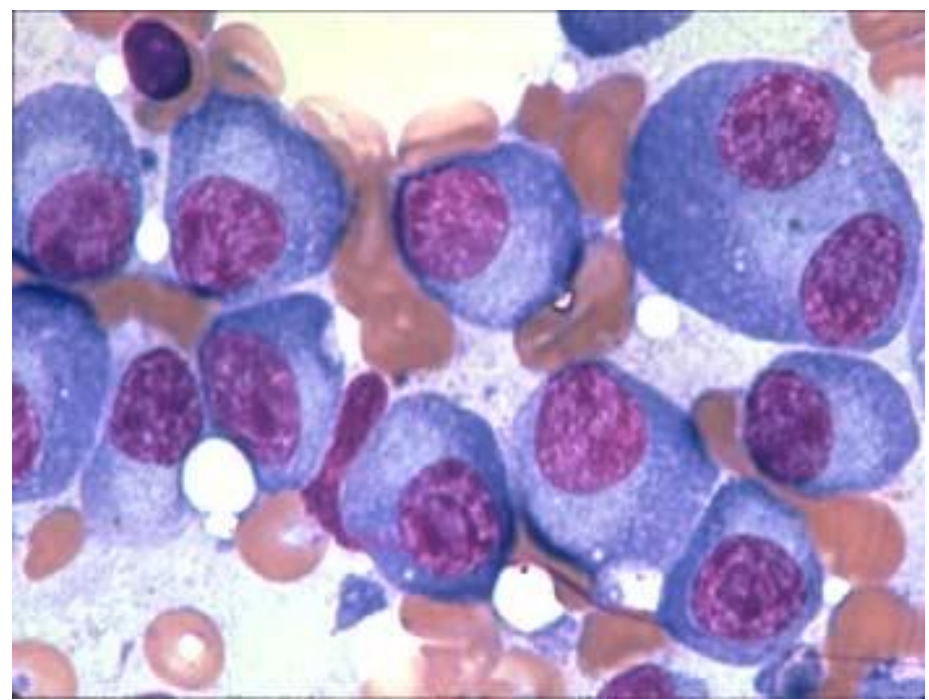

Figure 1: Bone Marrow Aspirate showing infiltration of bone marrow by $>10 \%$ plasma cells in a patient with smoldering myeloma

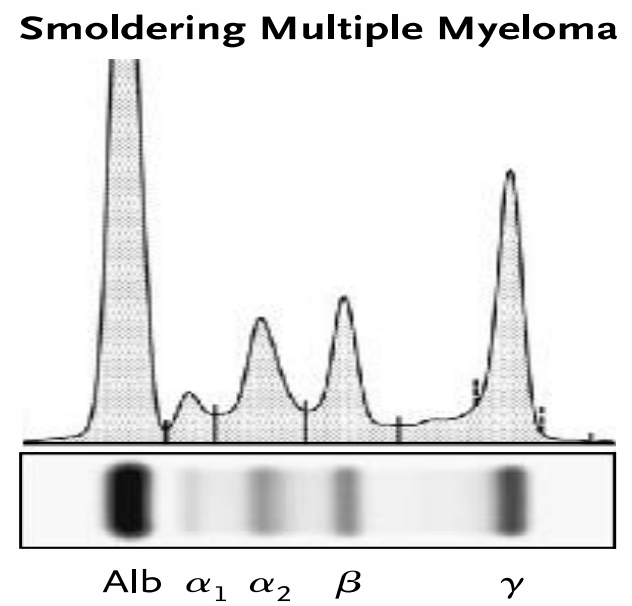

Figure 2: Serum protein electrophoresis showing $M$ protein $>3 \mathrm{gm} / \mathrm{dL}$ in a patient with smoldering myeloma 


\section{Defining risk in SMM:}

Many investigators have evaluated the risk of progression among patients with SMM. In most of the earlier studies, "high-risk" patients had annual progression rates as high as $25 \%$ to $40 \%$, depending on the risk criteria applied, and had survival rates comparable to patients with active myeloma [7] Risk factors include:

1) The number of bone lesions: this is the most commonly identified risk factor for progression to active myeloma in the era before the 2003 IMWG criteria [2,4,7-9]. The realization that patients with lytic lesions were among those with the shortest time to requiring systemic therapy contributed to the decision of excluding those patients with lytic bone lesions from the modern SMM definition.

2) The size of the $M$ spike and the degree of plasmacytosis were also consistent risk factors [10].

3) The $\lg A$ isotype $[11,12]$.

4) The presence of proteinuria [12,13].

5) An abnormal serum immunoglobulin free light chain (FLC) ratio [14-16]

6) Circulating plasma cells by slide-based immunofluorescence $[17,18]$

7) A high proliferative rate of bone marrow plasma cells (BMPCs) [19]

8) Immunoparesis [20].

9) A high percentage of BMPCs with aberrant flow cytometry $[16,20]$.

10) An abnormal magnetic resonance imaging (MRI) [16,21-23]. Among asymptomatic MM patients with normal radiographs, $50 \%$ have tumor-related abnormalities on MRI of the lower spine (Figure 3)[24].

11) Cytogenetics: The detection of deletion $17 p$ or $t(4 ; 14)$ by interphase fluorescence in situ hybridization (FISH) is associated with the shortest time to progression (TTP) and trisomies are a risk factor for progression from SMM to MM $[25,26]$. The Heidelberg group also found that gains of 1 q21 were a risk factor for progression among patients with SMM. These authors made an effort to relate FISH abnormalities with other reported risk factors, most notably tumor burden, and found that risk of the high-risk FISH was independent of tumor burden on multivariate analysis, with the greatest impact among those patients with lower tumor burden [26] There are fewer data about the risk of abnormal metaphase cytogenetics in SMM [27], in part because they are normal in $70 \%$ of patients with 
newly diagnosed MM; however, abnormal metaphase cytogenetics are a reflection of proliferative myeloma [28] and are also a risk factor for progression [29].

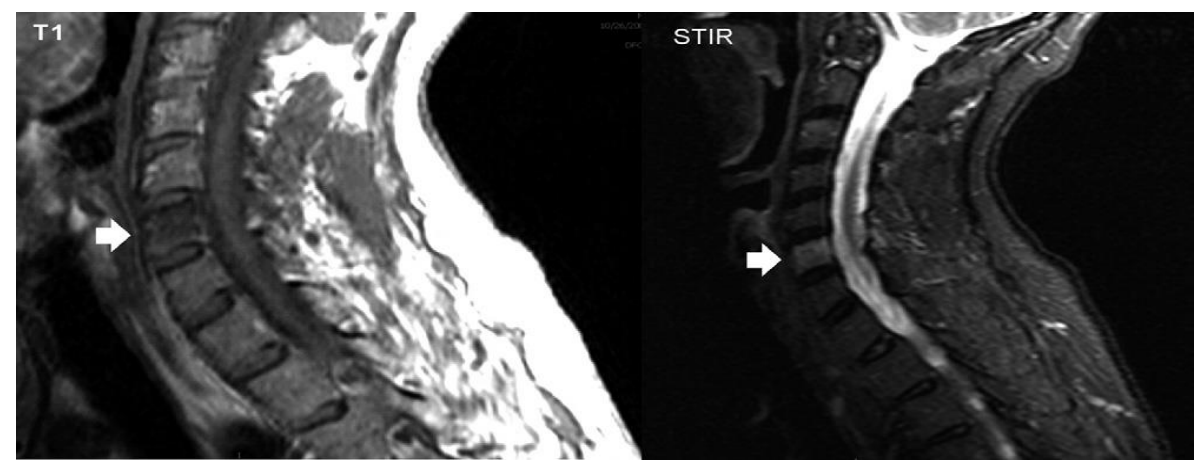

Figure 3: MRI of spine showing abnormality in a patient with smoldering myeloma and normal radiograph

\section{Results of interventional therapeutic trials:}

As mentioned, the purpose of the SMM construct was to bridge the gray zone between MGUS and MM. The separation was useful for management because SMM patients had a risk of progression many times greater than MGUS patients and hence needed more frequent follow-up than MGUS patients. Similarly, SMM patients were distinguished from MM because they could be observed without therapy until evidence of disease progression. This strategy was aimed at avoiding unnecessary side effects and cumulative exposure of alkylating drugs, which were found to be associated with myelodysplastic syndrome and acute leukemia [3033].

\section{Melphalan}

Two small randomized clinical trials were reported in the 1990s comparing immediate institution of melphalan and prednisone to initiation only once patients progressed from SMM to symptomatic MM. Neither of these trials demonstrated a survival advantage, although they were not adequately powered to make definitive conclusions [34-36].

\section{Bisphosphonates}

The next class of drug evaluated in SMM patients in prospective clinical trials was 
single-agent bisphosphonate [37-39]. Neither of the randomized trials demonstrated improved TTP or OS, but both demonstrated fewer skeletal related events (SREs) with bisphosphonate use.

\section{Thalidomide}

Thalidomide with or without bisphosphonate has been studied in patients with SMM in phase 2 trials and in 1 underpowered randomized controlled trial [40-44]. In the Mayo Clinic randomized controlled trial [44], there was a significant improvement in PFS in the thalidomide/zoledronic acid arm compared with the zoledronic acid alone arm (29 months vs 14 months) but no difference in PFS (49 months vs 40 months; $\mathrm{P}=.18$ ) or in OS (6-year OS > 70\%) [44]. The overall response rate was $37 \%$ for the thalidomide-containing arm and none with the zoledronic acid group. Thalidomide was poorly tolerated, with $80 \%$ of the thalidomide group developing grade 1 or 2 peripheral neuropathy and $74 \%$ with grade 1 or 2 fatigue in the thalidomide/zoledronic acid arm. The patients treated with zoledronic acid alone also had adverse effects, including grade 1 or 2 fatigue in $52 \%$ and grade 1 or 2 peripheral neuropathy in $18 \%$. The outcomes of this phase 3 trial differed slightly from its phase 2 predecessors in that TTP was shorter than that of Barlogie et al [40], or Rajkumar et al [41], (29 months vs 4-year event-free survival $60 \%$ vs 35 months, respectively). Part of the discrepancy may relate to the fact that the Barlogie et al study allowed for all-risk SMM patients and that the Rajkumar et al phase 3 study allowed for patients with IMM to enter. Another discrepancy between these studies is that patients in the Barlogie et al study who achieved a partial response or better had a shorter TTP than the non-responders, in contrast to the findings of the 2 Mayo-led trials. Indeed, Barlogie et al's study was concerning in that it implied that treatment with thalidomide may actually select for more aggressive myeloma clones to emerge under the selective pressure of the drug.

\section{Lenalidomide}

The most provocative study for patients with SMM is that of the PETHEMA-GEM (Programa Español de Tratamientos en Hematologica) group [45]. The authors reported on 119 patients with high-risk SMM managed in an open-label randomized controlled trial by either observation or lenalidomide and dexamethasone. Patients in the abstention arm were more likely to develop symptomatic disease ( $76 \%$ vs $23 \%)$. The overall response rate during induction therapy was $79 \%$, including $65 \%$ partial responses, $11 \%$ very good partial responses, $14 \%$ complete responses, and $7 \%$ stringent complete responses. In the treatment group, there were no grade 4 adverse events, but there was 1 death due to pneumonia and $12 \%$ of patients had serious adverse events as compared with $3 \%$ in the observation arm. With a median follow-up of 40 months, the treated patients had a superior 3-year survival without progression to symptomatic disease (77\% vs 30\%; $P<.001)$ and a superior 3 -year OS (94\% vs $80 \% ; P=.03$ ) from the time of registration. A major limitation in interpreting this study was the difference 
in how asymptomatic biochemical progression was handled in the 2 groups. In the observation arm, full CRAB progression was required for patients to receive antimyeloma therapy, whereas in the treatment arm, asymptomatic biochemical progression (> $25 \%$ increase of monoclonal component) during maintenance lenalidomide, was sufficient to warrant salvage with dexamethasone (or reescalation of lenalidomide). The design of the study do not allow to clearly determine if a preemptive strategy may be equally beneficial with less toxicity than a prophylactic strategy. Moreover, this difference in managing asymptomatic biochemical progression events may explain the relatively high 3-year mortality of $20 \%$ in the control arm. Another caveat is that lenalidomide-dexamethasone was not used consistently as salvage for the abstention group upon progression.

\section{Rethinking the definition of SMM and timing of therapy:}

Some have argued that SMM is not a unique biologic state, but rather a heterogeneous entity comprising some patients with biological premalignancy (MGUS) and some with true malignancy who have yet to declare clinical end-organ damage $[46,47]$ With the advent of multiple novel markers of disease (from MRI to positron emission tomography/computed tomography [PET/CT] to flow phenotype to FISH cytogenetics) and of newer (and presumably safer) antimyeloma therapies should the definition of SMM be reconsidered?

Patients who are considered to have the highest-risk SMM should be moved into the active MM category in order to preserve the doctrine that SMM is an entity that can be observed without therapy. The time has come when not treating a subset of what has up until now been considered high-risk SMM is more dangerous than treating. Bianchi and his colleagues have previously shown that even among patients with MGUS, the transition to MM can be unexpected and associated with end-organ damage in $40 \%$ of patients who do progress [48]. In other studies among patients with SMM who are observed until CRAB, the rates of renal failure were $11 \%$ to $13 \%$ and SREs $58 \%$ to $73 \%[7,38]$. In yet another study, $32 \%$ of the clinical progressions were severe as defined as the need for red blood cell transfusion, dialysis, or treatment of a pathological fracture [49]. Also worthy of consideration is the question of whether some of the lowest-risk SMM patients should be shifted into the MGUS category in order to reduce anxiety and intensity of follow-up, because the absence of risk factors predicts not only a longer TTP, but also a superior OS. To date, annual rates of progression in the "low-risk" SMM are reduced from approximately $10 \%$ per year to $3 \%$ to $5 \%$ per year. Although this is a significant reduction, these rates of progression are still slightly higher than that of high-risk MGUS [50].

As the questions about treating groups of SMM patients are considered, there must be agreement about acceptable rates of "overtreatment" and "undertreatment" of patients [51]. Most systems contain high-risk groups with 2-year TTP rates of $60 \%$. 
The 4 exceptions are bone marrow plasmacytosis of $>60 \%$ [16,52], serum immunoglobulin FLC ratio $>100[15,16]$, circulating plasma cells by slide-based immunofluorescence [18], and $>1$ focal lesion on whole-body MRI (Figure 4) [23]. Bone marrow plasmacytosis of $60 \%$ affects $2 \%$ to $8 \%$ of all SMM patients, yields a median TTP of 7 months to 15 months [16,52], and had a specificity of $95.5 \%$ for progression at 18 months [16]. The involved FLC/uninvolved FLC of 100 or greater captures approximately $7 \%$ to $15 \%$ of the SMM population and had a specificity of $98 \%$ for progression at 18 months [16]. With a median TTP of 13 to 15 months, a 2 -year TTP of $79 \%$, and a 5 -year TTP of $94 \%[15,16]$, shifting these populations into the active MM category would also be reasonable, though it would be of interest to know how many of these "high-risk" SMM had smoldering light chain myeloma [53]. More than 1 focal lesion on whole-body MRI, which affected $15 \%$ of SMM patients in one study, had a high predictive value for progression to active MM with a median TTP of 13 months and a 2-year TTP of $70 \%$ [23]. Diffuse marrow infiltration pattern was also significant on multivariate analysis. Circulating plasma cells as detected by slide-based immunofluorescence captures $15 \%$ of SMM patients and yielded a median TTP of 12 months [18], but this test is not readily available. Patients with high-risk FISH [deletion 17p, t(4;14), and gain 1q21] might be considered as active myeloma and be candidates for early treatment, but these groups are too heterogeneous to make that recommendation [6].

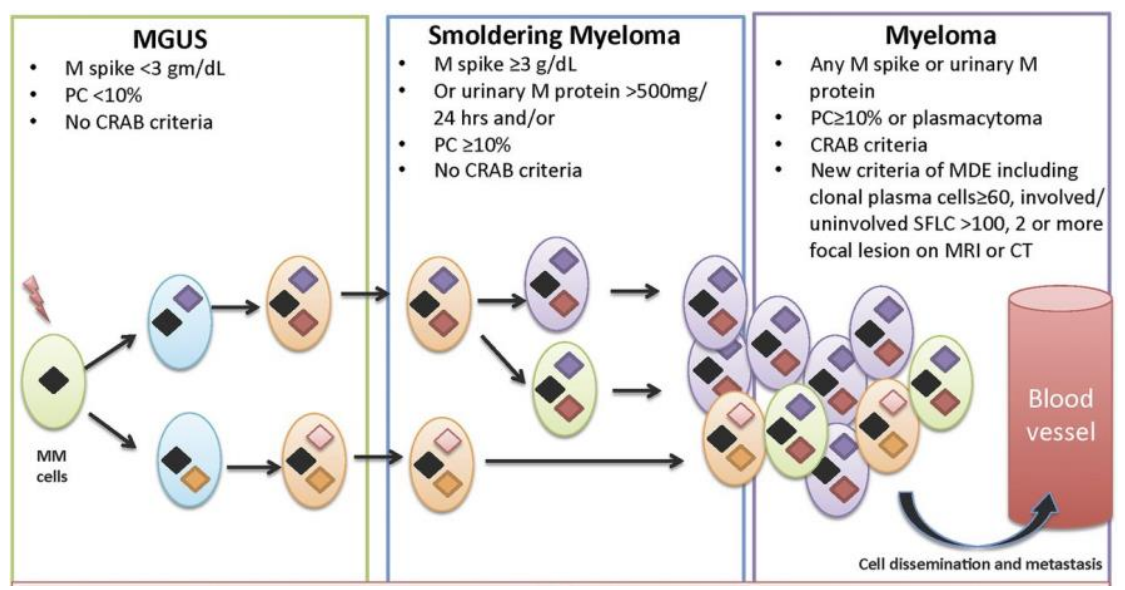

Figure 4: New definitions of MGUS, smoldering myeloma and MM requiring therapy

\section{Consensus recommendations on treatment:}

It is possible, but not proven, that some of the improved survival seen in epidemiologic studies [54-56], may be in part a function of physicians being more willing to treat patients earlier, which potentially exaggerates the beneficial impact 
of novel therapies over the past 15 years. The question remains, however, whether treating sooner than later improves quality of life and/or OS. Observation as practiced in the PETHEMA-GEM study in patients with "high-risk" SMM was associated with an unacceptable early mortality that was significantly decreased by early treatment with lenalidomide and dexamethasone [45]. As mentioned earlier, some of the benefit (both survival and time to symptomatic disease) observed in this study may relate to the protocol design: treating biochemical progression and a 30\% drop-out rate by "choice" in the treatment arm and strict adherence fulfilling a CRAB criterion prior to instituting therapy in the observation arm. There are additional caveats that limit the generalizability of the PETHEMAGEM study. First, the trial results apply not to all patients with SMM, but only to "high-risk" SMM patients as defined by the trial criteria. Forty percent of patients enrolled did so purely based on the flow-based definition of plasma cell immunophenotype, a methodology that is not available in most institutions and that requires considerable expertise to interpret the results even if the technology were available. Second, the authors did not use lenalidomide-dexamethasone as salvage for the abstention trial universally. Third, this strategy may result in overtreatment of approximately $40 \%$ of patients at 3 years, $30 \%$ of patients at 4 years, and $20 \%$ of patients at 5 years. Fourth, the costs of intervention also need to be considered [51]. Although the "cost" of undertreatment is partially captured (more bone lesions and renal failure and now a suggestion of inferior OS), the "cost" of overtreatment is less clear. With the simplest of regimens (i.e., lenalidomide plus dexamethasone), the annual cost of therapy is approximately 100000 USD, not including the extra monitoring required for patients on active therapy and management of adverse events [57,58]. Part of the "cost" of overtreatment may include increased side effects, which may translate into inferior quality of life. Finally, long-term safety data for protracted lenalidomide use are limited. The potential of this last "cost" would be abrogated if physicians choose to treat according to the method of the PETHEMA-GEM study (i.e., only 2 years of lenalidomide followed by observation until progression), a practice gaining favor in light of concerns of the potential risk of cumulative risk of secondary malignancy [59-61].

After reviewing all of the data, taking into account the risks and benefits of observation as well as the risks and benefits of intervention, some recommendations for the management of SMM patients have been suggested. Clearly, there is still room for finding better predictors, but for now it is recommended to change the definition of active $\mathrm{MM}$, in the absence of CRAB, to include (1) patients with bone marrow plasmacytosis $\geq 60 \%$, (2) a ratio of involved to uninvolved FLC of $\geq 100$, or (3) whole-body MRI demonstrating $>1$ focal lesion. In these patients, the risk of progression in the first 2 or 3 years is $80 \%$ or higher. Once defined as having active $\mathrm{MM}$, these patients should receive therapy appropriate for any newly diagnosed patient, and one such therapy now supported with phase 3 evidence would be lenalidomide plus dexamethasone as used in the 
PETHEMA-GEM interventional arm, though the combination is not Food and Drug Administration approved as first-line therapy in the United States [6].

The cost of performing whole-body MRI on all patients with SMM with the intent of treating only those with $>1$ focal lesion on MRI would be much less expensive than treating a SMM patient who did not require treatment of 2 years or more. Limitations to using whole-body MRI are that many institutions do not have an algorithm to perform or interpret the test and that payment for the test may not be reimbursed by insurance providers. PET/CT may be a nice alternative to wholebody MRI because PET/CT has a superior sensitivity to standard bone radiographs, is faster and more comfortable for the patient, and can be used in patients with implanted pacemakers and defibrillators [62-64].

It is recommended that all other patients with SMM be observed without therapy every 3 to 6 months and encouraged to participate in clinical trials. Although the PETHEMA-GEM trial shows that a subset of these patients (those with both BMPCs > 10\% and M protein > $30 \mathrm{~g} / \mathrm{L}$ ) could benefit from therapy, it is not recommended to intervene at this point until further confirmatory evidence emerges, though it is important that these data be shared with patients. These recommendations should be considered by the IMWG to arrive at an international consensus (Figure 5).

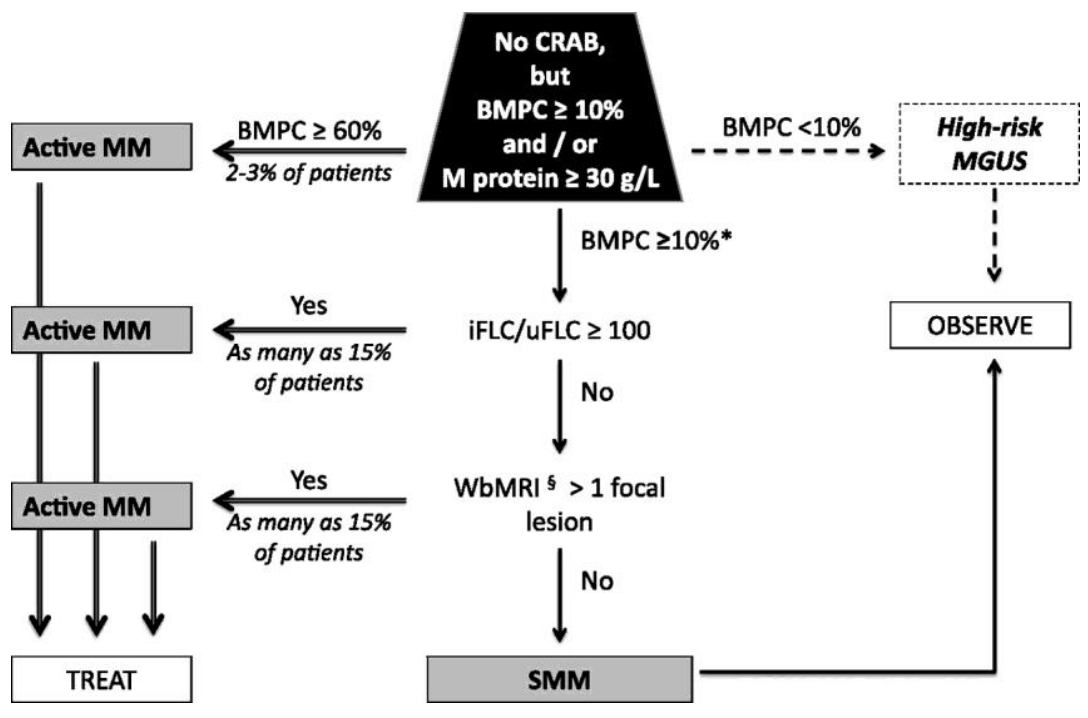

Figure 5: Algorithm for reclassifying SMM and active MM. *Consider including patients with the following FISH: deletion 17p, t(4;14), and 1q21 gains as active $\mathrm{MM}$; this population could account for as many as $30 \%$ of SMM patients. §Consider using more than 1 fluorodeoxyglucose-avid lesion on PET/CT 
in lieu of MRI. iFLC, involved FLC; uFLC, uninvolved FLC; WbMRI, whole-body MRI [6].

\section{References:}

1. Kyle RA, Greipp PR. Smoldering multiple myeloma. N Engl J Med. 1980;302(24):1347-1349.

2. International Myeloma Working Group. Criteria for the classification of monoclonal gammopathies, multiple myeloma and related disorders: a report of the International Myeloma Working Group. Br J Haematol. 2003;121(5):749-757.

3. Alexanian R. Localized and indolent myeloma. Blood. 1980;56(3):521525.

4. Alexanian R, Barlogie B, Dixon D. Prognosis of asymptomatic multiple myeloma. Arch Intern Med. 1988;148(9):1963-1965.

5. Rosinol L, Blade J, Esteve J, et al. Smoldering multiple myeloma: natural history and recognition of an evolving type. $\mathrm{Br} J$ Haematol. 2003;123(4):631-636.

6. Dispenzieri A, Stewart AK, Chanan-Khan A, et al. Smoldering multiple myeloma requiring treatment: time for a new definition? Blood. 2013;122(26):4172-4181.

7. Wisloff $F$, Andersen $P$, Andersson TR, et al. Incidence and follow-up of asymptomatic multiple myeloma. The myeloma project of health region I in Norway. II. Eur J Haematol. 1991;47(5):338-341.

8. Durie BG, Salmon SE, Moon TE. Pretreatment tumor mass, cell kinetics, and prognosis in multiple myeloma. Blood. 1980;55(3):364-372.

9. Durie BG, Salmon SE. A clinical staging system for multiple myeloma. Correlation of measured myeloma cell mass with presenting clinical features, response to treatment, and survival. Cancer. 1975;36(3):842854.

10. Kyle RA, Remstein ED, Therneau TM, et al. Clinical course and prognosis of smoldering (asymptomatic) multiple myeloma. N Engl J Med. 2007;356(25):25822590.

11. Facon T, Menard JF, Michaux JL, et al. Prognostic factors in low tumour mass asymptomatic multiple myeloma: a report on 91 patients. The Groupe d'Etudes et de Recherche sur le Myelome (GERM). Am J Hematol. 1995;48(2):71-75.

12. Weber DM, Dimopoulos MA, Moulopoulos LA, Delasalle KB, Smith T, Alexanian R. Prognostic features of asymptomatic multiple myeloma. $\mathrm{Br}$ J Haematol. 1997;97(4):810-814.

13. Dimopoulos MA, Moulopoulos A, Smith T, Delasalle KB, Alexanian R. Risk 
progression in asymptomatic multiple myeloma. Am $\mathrm{J}$ Med. 1993;94(1):57-61.

14. Dispenzieri A, Kyle RA, Katzmann JA, et al. Immunoglobulin free light chain ratio is

an independent risk factor for progression of smoldering (asymptomatic) multiple myeloma. Blood. 2008;111(2):785-789.

15. Larsen JT, Kumar SK, Dispenzieri A, Kyle RA, Katzmann JA, Rajkumar $\mathrm{SV}$. Serum free light chain ratio as a biomarker for high-risk smoldering multiple myeloma. Leukemia. 2013;27(4):941-946.

16. Kastritis E, Terpos E, Moulopoulos L, et al. Extensive bone marrow infiltration and abnormal free light chain ratio identifies patients with asymptomatic myeloma at high risk for progression to symptomatic disease. Leukemia. 2013;27(4):947-953.

17. Witzig TE, Kyle RA, O'Fallon WM, Greipp PR. Detection of peripheral blood plasma cells as a predictor of disease course in patients with smouldering multiple myeloma. Br J Haematol.1994;87(2):266-272.

18. Bianchi G, Kyle RA, Larson DR, et al. High levels of peripheral blood circulating plasma cells as a specific risk factor for progression of smoldering multiple myeloma. Leukemia. 2013;27(3):680-685.

19. Madan S, Kyle RA, Greipp PR. Plasma cell labeling index in the evaluation of smoldering (asymptomatic) multiple myeloma. Mayo Clin Proc. 2010;85(3):300.

20. Perez-Persona E, Vidriales MB, Mateo G, et al. New criteria to identify risk of progression in monoclonal gammopathy of uncertain significance and smoldering multiple myeloma based on multiparameter flow cytometry analysis of bone marrow plasma cells. Blood. 2007;110(7):2586-2592.

21. Mariette $X$, Zagdanski AM, Guermazi $A$, et al. Prognostic value of vertebral lesions detected by magnetic resonance imaging in patients with stage I multiple myeloma. Br J Haematol. 1999;104(4):723-729.

22. Moulopoulos LA, Dimopoulos MA, Smith TL, et al. Prognostic significance of magnetic resonance imaging in patients with asymptomatic multiple myeloma. J Clin Oncol. 1995;13(1):251-256.

23. Hillengass J, Fechtner K, Weber MA, et al. Prognostic significance of focal lesions in whole body magnetic resonance imaging in patients with asymptomatic multiple myeloma. J Clin Oncol. 2010;28(9):1606-1610.

24. Pertuiset E, Bellaiche L, Liote F, Laredo JD. Magnetic resonance imaging of the spine in plasma cell dyscrasias. A review. Rev Rhum Engl Ed. 1996;63(11):837845.

25. Rajkumar SV, Gupta V, Fonseca R, et al. Impact of primary molecular cytogenetic abnormalities and risk of progression in smoldering multiple myeloma. Leukemia. 2013;27(8):1738-1744.

26. Neben K, Jauch A, Hielscher T, et al. Progression in smoldering myeloma is independently determined by the chromosomal abnormalities del(17p), 
$\mathrm{t}(4 ; 14)$, gain 1q, hyperdiploidy, and tumor load [published online ahead of print October 21, 2013]. J Clin Oncol. DOI:10.1200/JCO.2012.48.4923.

27. Lloveras E, Sole F, Florensa L, et al. Contribution of cytogenetics and in situ hybridization to the study of monoclonal gammopathies of undetermined significance. Cancer Genet Cytogenet. 2002;132(1):25-29.

28. Rajkumar S, Fonseca R, Lacy M, et al. Abnormal cytogenetics predict poor survival after high-dose therapy and autologous blood cell transplantation in multiple myeloma. Bone Marrow Transplant. 1999;24(5):497-503.

29. Depil S, Leleu X, Micol JB, et al. Abnormal cytogenetics and significant bone

marrow plasmacytosis are predictive of early progression and short survival in patients with low tumor mass asymptomatic multiple myeloma. Leuk Lymphoma. 2004;45(12):2481-2484.

30. Kyle RA, Pierre RV, Bayrd ED. Multiple myeloma and acute myelomonocytic leukemia. N Engl J Med. 1970;283(21):1121-1125.

31. Rosner $F$, Grunwald $H$. Multiple myeloma terminating in acute leukemia. Report of 12 cases and review of the literature. Am $\mathrm{J}$ Med. 1974;57(6):927-939.

32. Kyle RA, Pierre RV, Bayrd ED. Multiple myeloma and acute leukemia associated with alkylating agents. Arch Intern Med. 1975;135(1):185-192.

33. Bergsagel DE. Chemotherapy of myeloma: drug combinations versus single agents, an overview, and comments on acute leukemia in myeloma. Hematol Oncol. 1988;6(2):159-166.

34. Hjorth M, Hellquist L, Holmberg E, Magnusson B, Rodjer S, Westin J; Myeloma Group of Western Sweden. Initial versus deferred melphalan prednisone therapy for asymptomatic multiple myeloma stage $\mathrm{I}-\mathrm{a}$ randomized study. Eur J Haematol. 1993;50(2):95-102.

35. Riccardi A, Ucci G, Luoni R, et al; Cooperative Group of Study and Treatment of Multiple Myeloma. Treatment of multiple myeloma according to the extension of the disease: a prospective, randomised study comparing a less with a more aggressive cystostatic policy. $\mathrm{Br} \mathrm{J}$ Cancer. 1994;70(6):1203-1210.

36. Riccardi A, Mora O, Tinelli C, et al; Cooperative Group of Study and Treatment of Multiple Myeloma. Long-term survival of stage I multiple myeloma given chemotherapy just after diagnosis or at progression of the disease: a multicenter randomized study. Br J Cancer. 2000;82(7):12541260.

37. Musto P, Falcone A, Sanpaolo G, et al. Pamidronate reduces skeletal events but does not improve progression-free survival in early stage untreated myeloma: results a randomized trial. Leuk Lymphoma. 2003; 44(9):1545-1548.

38. D'Arena G, Gobbi PG, Broglia C, et al; Gimema (Gruppo Italiano Malattie Ematologiche

Dell'Adulto); Multiple Myeloma Working Party; Gisl (Gruppo Italiano 
Studio Linfomi) Cooperative Group. Pamidronate versus observation in asymptomatic myeloma: final results with longterm follow-up of a randomized study. Leuk Lymphoma. 2011;52(5):771-775.

39. Musto P, Petrucci MT, Bringhen S, et al; GIMEMA (Italian Group for Adult Hematologic Diseases)/Multiple Myeloma Working Party and the Italian Myeloma Network. A multicenter, randomized clinical trial comparing zoledronic acid versus observation in patients with asymptomatic myeloma. Cancer. 2008;113(7):1588-1595.

40. Barlogie B, van Rhee F, Shaughnessy JD Jr, et al. Seven-year median time to progression with thalidomide for smoldering myeloma: partial response identifies subset requiring earlier salvage therapy for symptomatic disease. Blood. 2008;112(8):3122-3125.

41. Rajkumar SV, Dispenzieri A, Fonseca R, et al. Thalidomide for previously untreated indolent or smoldering multiple myeloma. Leukemia. 2001;15(8):1274-1276.

42. Detweiler-Short K, Hayman S, Gertz MA, et al. Long-term results of single-agent thalidomide as initial therapy for asymptomatic (smoldering or indolent) myeloma. Am J Hematol. 2010;85(10):737-740.

43. Weber D, Rankin K, Gavino M, Delasalle K, Alexanian R. Thalidomide alone or with dexamethasone for previously untreated multiple myeloma. J Clin Oncol. 2003;21(1):16-19.

44. Witzig TE, Laumann KM, Lacy $M Q$, et al. A phase III randomized trial of thalidomide plus zoledronic acid versus zoledronic acid alone in patients with asymptomatic multiple myeloma. Leukemia. 2013;27(1):220-225.

45. Mateos MV, Hernandez MT, Giraldo P, et al. Lenalidomide plus dexamethasone for high-risk smoldering multiple myeloma. $N$ Engl J Med. 2013;369(5):438-447.

46. Rajkumar SV. Prevention of progression in monoclonal gammopathy of undetermined significance. Clin Cancer Res. 2009;15(18):5606-5608.

47. Rajkumar SV. Treatment of multiple myeloma. Nat Rev Clin Oncol. $2011 ; 8(8): 479-491$.

48. Bianchi G, Kyle RA, Colby CL, et al. Impact of optimal follow-up of monoclonal gammopathy of undetermined significance on early diagnosis and prevention of myeloma-related complications. Blood. 2010;116(12):2019-2025, quiz 2197.

49. Rago A, Grammatico S, Za T, et al; Multiple Myeloma GIMEMA-Latium Region Working Group. Prognostic factors associated with progression of smoldering multiple myeloma to symptomatic form. Cancer. 2012;118(22):5544-5549.

50. Rajkumar SV, Kyle RA, Therneau TM, et al. Serum free light chain ratio is an independent risk factor for progression in monoclonal gammopathy of undetermined significance. Blood. 2005;106(3):812-817.

51. Kyle RA. Role of maintenance therapy after autologous stem cell transplant 
myeloma: lessons for cancer therapy. Mayo Clinic Proc. 2011;86(5):419420.

52. Rajkumar SV, Larson D, Kyle RA. Diagnosis of smoldering multiple myeloma. N Engl J Med. 2011;365(5):474-475.

53. Kyle RA, Larson D, Therneau TM, et al. Idiopathic bence jones proteinuria (smoldering monoclonal light-chain proteinuria): clinical course and prognosis. [abstract]. ASH Annual Meeting Abstracts. 2012;120(21):1861.

54. Pulte D, Gondos A, Brenner H. Improvement in survival of older adults with multiple myeloma: results of an updated period analysis of SEER data. Oncologist. 2011;16(11):1600-1603.

55. Kumar SK, Rajkumar SV, Dispenzieri A, et al. Improved survival in multiple myeloma and the impact of novel therapies. Blood. 2008;111(5):2516-2520.

56. Brenner H, Gondos A, Pulte D. Recent major improvement in long-term survival of younger patients with multiple myeloma. Blood. 2008;111(5):2521-2526.

57. Bonkowski J, Vermeulen LC, Kolesar JM. The clinical utility of lenalidomide in multiple myeloma and myelodysplastic syndromes. J Oncol Pharm Practice. 2010;16(4):223-232.

58. Durie B, Binder G, Pashos C, Khan Z, Hussein M, Borrello I. Total cost comparison in relapsed/refractory multiple myeloma. J Media Econ. 2013;16(5):614-622.

59. Usmani SZ, Sexton R, Hoering A, et al. Second malignancies in total therapy 2 and 3 for newly diagnosed multiple myeloma: influence of thalidomide and lenalidomide during maintenance. Blood. 2012;120(8):1597-1600.

60. Attal M, Lauwers-Cances V, Marit G, et al; IFM Investigators. Lenalidomide maintenance after stem-cell transplantation for multiple myeloma. N Engl J Med. 2012;366(19):1782-1791.

61. McCarthy PL, Owzar K, Hofmeister CC, et al. Lenalidomide after stemcell transplantation for multiple myeloma. $\mathrm{N}$ Engl $\mathrm{J}$ Med. 2012;366(19):1770-1781.

62. Bodet-Milin C, Eugene T, Bailly C, et al. FDG-PET in the evaluation of myeloma in 2012. Diagn Interv Imaging. 2012;S2211-5684(12)00401-9.

63. Spinnato $P$, Bazzocchi A, Brioli A, et al. Contrast enhanced MRI and 18FFDG PET-CT in the assessment of multiple myeloma: a comparison of results in different phases of the disease. Eur J Radiol. 2012;81(12):40134018.

64. Walker RC, Brown TL, Jones-Jackson LB, De Blanche L, Bartel T. Imaging of multiple myeloma and related plasma cell dyscrasias. J Nucl Med. 2012;53(7):1091-1101. 

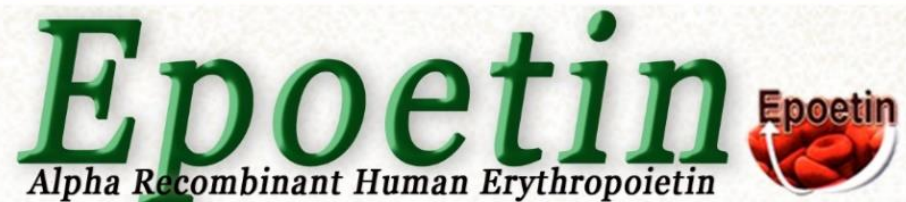
2000 or 4000 IU

\section{Anemia...}

A tough Problem With an Easy Approach

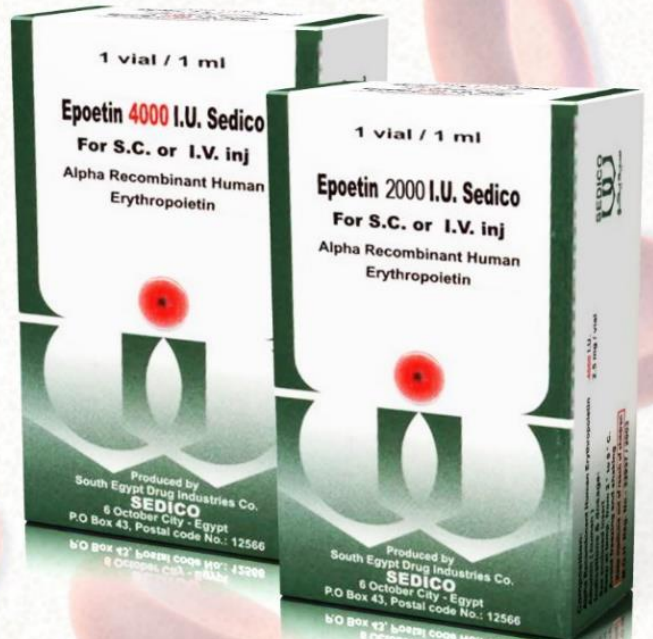

- Effective control of Anemia

Oncreased feeling of well being

-High safety profile

-High cost- effectiveness

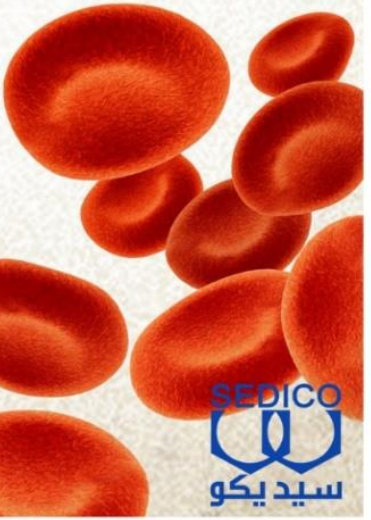

